not only an inflammatory factor but also an apolipoprotein that can replace apolipoprotein $\mathrm{A} 1$ (apoA1) as the major apolipoprotein of HDL. However, the relationship between genetic polymorphisms of $\mathrm{SAA}$ and $\mathrm{CAD}$ remains unclear.

Methods 4 Single Nucleotide Polymorphisms (SNPs) (rs12218, rs1059559, rs2229338, and rs2468844) of SAA1 and SAA2 gene were genotyped in $1580 \mathrm{CAD}$ patients and 1914 age- and sex-matched controls by the use of PCR-restriction fragment length polymorphism (PCR-RFLP) analysis.

Results The CC genotype and C allele of rs12218 and the GG genotype and $\mathrm{G}$ allele of rs2468844 were more common in the CAD patients than in the control subjects, respectively (all $\mathrm{p}<0.001$ ). After adjusted for diabetes mellitus, hypertension, smoking, drinking and lipid disorders by use of logistic regression, the SNPs rs12218 (OR=5.906, 95\% CI 2.877 to $12.124, p<0.001)$ and rs2468844 (OR=4.102, 95\% CI 2.018 to 8.129 , p < 0.001) still differed significantly between the CAD patients and control subjects.

Conclusion These data suggest that genetic polymorphisms of SAA1/2 gene significantly increased the risk of CAD in a Chinese Han population.

\section{e0082 MOLECULAR IMAGING OF APELIN ON SURVIVAL AND FUNCTION OF MESENCHYMAL STEM CELLS IN HINDLIMB MICE}

doi:10.1136/hrt.2010.208967.82

Dong Liang, Weijie Li, Shuang Li, Rongqing Zhang, Lina Gao, Shunming Zhu, Yabin Wang, Haichang Wang, Feng Cao. Department of Cardiology, Xijing Hospital, Fourth Military Medical University, Xi'an, Shaanxi, China

Objective This study was designed to evaluate the contribution of apelin to the therapeutic efficacy of mesenchymal stem cells in hindlimb ischaemia mice.

Methods Mesenchymal stem cells (MSC) expressing firefly luciferase (Fluc) were isolated from B-actin-luc mice and characterised by flow cytometry and bioluminescence imaging (BLI). Male FVB mice underwent femoral artery ligation and received MSC $\left(1 \times 10^{6}\right)$ or MSC with Apelin intra-quadriceps femoris muscle injection. Cell survival was imaged by BLI. Angiogenesis was assessed by immunohistochemisty method. The expressions of AKT and pAKT after cellular therapy were analysed by Western blot.

Results Fluc expression correlated with cell number in all groups. In vivo BLI revealed acute donor cell death of MSC within 2 weeks after transplantation. By contrast, signals of injected cells were still present after 4 weeks in the MSC with apelin group. Immunohistochemisty showed more angiogenesis in the MSC with Apelin group compared to MSC $(p<0.05)$. In vitro apelin treatment of MSC exposed to hypoxia increased cell proliferation. Moreover, considerable increases in phosphorylation of Akt were found in MSC pretreated with apelin.

Conclusions Apelin has beneficial effects on the therapeutic efficacy and survival maintenance of mesenchymal stem cells in hindlimb ischaemia and might constitute an important therapy target in cardiovascular disease.

\section{e0083 MEMBRANE TYPE 1 MATRIX METALLOPROTEINASE ACTIVATION IS ENHANCED BY LOW SHEAR STRESS THROUGH INTEGRIN PATHWAY IN APOE-/- MICE}

\section{doi:10.1136/hrt.2010.208967.83}

Chen Liang, Cai Xiaojun, Li Xuan, Liu Xiaoling, Zhang Yun, Zhang Mei. Shandong University Qilu Hospital

Objective Low shear stress and matrix metalloproteinase are involved in atherogenesis and plaque stability. The aim of this study wad to explore the relation and possible mechanism of membrane type-1 matrix metalloproteinase (MT1-MMP) and low shear stress (LSS).

Methods 80 male apoE-/- mice were fed a high-fat diet, and lesions in the left carotid artery were induced by perivascular placement of constrictive collars.

Results Ultrasound-determined shear stress was significantly lower in the left carotid artery than in the right artery $(5.61 \pm 0.72$ vs $\left.9.76 \pm 1.48 \mathrm{~N} / \mathrm{m}^{2}, \mathrm{p}<0.01\right)$. Confocal microscopy and dual staining revealed higher MT1-MMP expression in left common carotid artery. Cultured human umbilical vein endothelial cells (HUVECs) exposed to $0.4 \mathrm{~N} / \mathrm{cm}^{2}$ shear stress (LSS) and $1.2 \mathrm{~N} / \mathrm{cm}^{2}$ shear stress (PSS). HUVECs subjected to LSS showed a time-dependent increase in MT1-MMP mRNA level. MT1-MMP mRNA level was downregulated under PSS at 3 and $5 \mathrm{~h}$. MT1-MMP protein showed no change in expression at 1-h LSS, but 3-, 5-, 8- and 16-h treatment produced significantly increased expression and activity. NF- $\kappa \mathrm{B}$ DNA-binding activity was stronger at 30-min and 1-h LSS exposure than with control treatment. Pretreatment with $18 \mu \mathrm{M}$ SN50 efficiently inhibited NF- $\kappa B$ DNA binding activity. In the presence of SN50, MT1-MMP mRNA expression, protein level and activity were significantly attenuated (all $\mathrm{p}<0.01$ ). MT1-MMP mRNA expression, protein level and activity were inhibited by PD98059 (all $p<0.01$ ). To determine the relation between ERK1/2 and NF-KB with MT1MMP induced by LSS, HUVECs were incubated with PD98059 for $2 \mathrm{~h}$ before LSS. The level of phosphor-IKK $\alpha / \beta$ was reduced, whereas that of phosphor-I $\mathrm{B} \alpha \boldsymbol{\alpha}$ was increased as compared with no PD98059 treatment. As well, NF- $\mathrm{BB}$ DNA-binding activity was decreased. HUVECs were preincubated for $48 \mathrm{~h}$ with FAK siRNA and then treated with LSS. The shear stress-induced increase in MT1-MMP mRNA and protein level and activity was significantly inhibited (all $\mathrm{p}<0.01)$. As well, LSS-induced ERK1/2 activation was inhibited with 5 -min LSS exposure $(\mathrm{p}<0.01)$. To examine NF- $\kappa B$ DNA-binding activity, HUVECs were pretreated for $48 \mathrm{~h}$ with FAK siRNA before

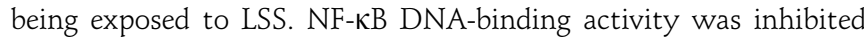
with FAK siRNA pretreatment. HUVECs were preincubated for $48 \mathrm{~h}$ with integrin $\beta 1$ siRNA and then underwent LSS. The shear stress-induced increase in MT1-MMP mRNA expression, protein level and activity was significantly inhibited (all $\mathrm{p}<0.01$ ). As well, LSS-induced FAK and ERK1/2 activation was inhibited at 5-min

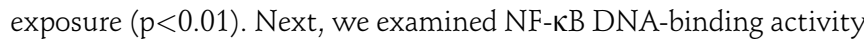
with 48-h integrin $\beta 1$ siRNA pretreatment before exposure to LSS; NF- $\kappa B$ DNA binding activity was inhibited by siRNA integrin $\beta 1$ pretreatment.

Conclusions MT1-MMP induced by LSS is involved in atherosclerotic plaque, via the integrin $\beta 1$-FAK-ERK1/2-NF-KB pathway.

\section{e0084 IMAGING OF VULNERABLE PLAQUE AND THROMBOSIS WITH MRI IN A RABBIT MODEL}

doi:10.1136/hrt.2010.208967.84

Zhao Quanming, Zeng Conghe, Feng Tingting, Zhao Xin, Ma Xiaohai, Zhang Zhaoqi. Beijing Anzhen Hospital

Objective Our aim is to investigate the feasibility of detecting vulnerable plaque and thrombosis by use of MRI.

Methods 24 male New Zealand White rabbits were divided into two groups: the atherosclerosis group (As group, $n=20$ ) and the normal control group ( $C$ group, $n=4$ ). After induction of atherosclerosis, MRI exams were conducted separately before and after the induction, triggering the plagues' disruption. The rabbits were then massacred to obtain data of pathology. The animals in the normal control group were fed a standard diet, and we performed MRI exam separately before and after, triggering the plagues' disruption too. After MRI exams, rabbits were massacred to obtain data of pathology. The in vivo imaging results of MRI were compared with the 